\title{
Optimal Planning of Active Distribution Network Based on Soft Open Point and Shunt Capacitors
}

\author{
Haiyue Yang ${ }^{1}, \mathrm{Lu} \mathrm{Yu}^{2 *}$, Qianyu Zhao ${ }^{2}$, Hu Wang ${ }^{1}$, Zongwei Du ${ }^{1}$, Bai Jie ${ }^{3}$ \\ ${ }^{1}$ State Grid Hengshui Electric Supply Company, Hengshui, Hebei, 053000, China \\ ${ }^{2}$ School of Electrical and Information Engineering, Tianjin University, Tianjin, 300072, China \\ ${ }^{3}$ Sunshine New Energy Development Co., Ltd, Hefei, Anhui, 230000, China
}

\begin{abstract}
As the penetration of distributed wind power in the distribution network continues to increase, the uncertainty of its output has a serious impact on the stable operation of the distribution network. It is difficult to meet the voltage regulation requirements when the wind power fluctuates frequently only by relying on shunt capacitors. Therefore, a coordinated optimization planning method based on soft open point (SOP) and shunt capacitors is proposed. Firstly, the bidirectional generative adversarial network (BIGAN) is used to characterize the uncertainty of wind power output and generate typical scenarios of wind power output. Secondly, a multi-objective optimization planning model of SOP and shunt capacitors is proposed based on the scene analysis method; Then, a solution strategy based on the improved elitist non-dominated sorting genetic algorithm (NSGA-II) is proposed. Finally, the proposed planning model and solution are verified and analyzed in the improved IEEE 33-bus system.
\end{abstract}

\section{Introduction}

Wind energy is a kind of renewable energy, which is widely distributed and non-emission. The integration of wind power is of great significance to accelerate energy transformation and implement sustainable development strategy for distribution network. However, the uncertainty of wind power output also brings great challenges to the stable operation and economic dispatch of distribution network. As a common reactive power compensation device, shunt capacitor plays an important role in dealing with the adverse effects of wind power access, but it is difficult to meet the voltage regulation requirements of wind power and load frequent

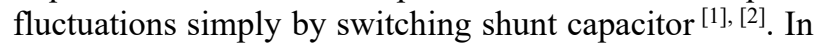
recent years, as a new type of flexible distribution equipment, intelligent soft open point (SOP) plays an important role in continuous power flow regulation and promoting wind power consumption. However, the investment and operation cost of SOP with precision function is high, and it is impossible to completely replace the traditional control means in the short term. Therefore, the coordination of SOP and shunt capacitor should be considered in the optimal planning of distribution network.

At present, a large number of researches have considered the influence of wind power output uncertainty in distribution network optimization planning. The scenario analysis method discretizes the random vectors with continuous probability distribution into a set of scenarios, which reduces the difficulty of solving the problem and ensures the economy of the planning scheme and the safety of the system operation. But the effect of optimal planning depends on the quality of scene generation. The existing scene generation methods are roughly divided into two types, which are based on statistical model or generation mode ${ }^{[3]}$. Among them, the latter is more widely used in scene generation by observing the existing samples, autonomously learning their distribution and generating similar samples ${ }^{[4]}$.

In order to further determine the objectives of optimization planning of distribution network, many objective functions are considered and a multi-objective planning model is established, which is now widely used. For multi-objective optimization problems, the solution methods include conventional algorithm and multiobjective evolutionary algorithm ${ }^{[5]}$. Among them, the latter can not only meet the needs of multi-objective parallel processing, but also has good convergence effect and fast calculation speed, which has been widely used to solve complex nonlinear multi-objective optimization problems.

In this paper, aiming at the cooperative planning of SOP and shunt capacitor, this paper firstly constructs a typical scenario of wind power output based on the scenario generation technology of bidirectional general adverse network (BIGAN). Secondly, considering the influence of the uncertainty of wind power output on the distribution network, a multi-objective optimal planning model of SOP and shunt capacitor is proposed based on scenario analysis method based on improved NSGA-II algorithm. Finally, the proposed model and solution method are verified in the improved IEEE 33 bus system.

"Corresponding author's e-mail: andy_yulu@tju.edu.cn 


\section{Modelling of wind power scenario generation method}

\subsection{Basic principle of BIGAN}

GAN is composed of generator and discriminator. The core idea is to set up a zero-sum game to learn the probability distribution of data through the confrontation of generator and discriminator. BIGAN is an improvement of GAN, which can map real data $\mathrm{X}$ to hidden variable space to realize feature extraction of real data. The principle is similar to GAN, but an encoder is added to the topology. The specific structure is shown in Figure 1.

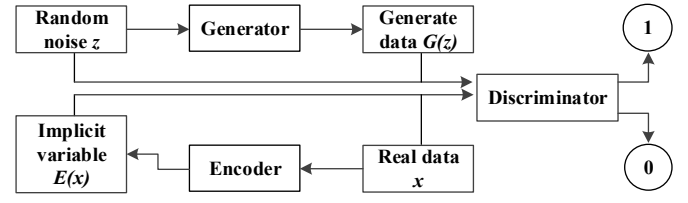

Figure 1. Schematic of BIGAN architecture

The optimization idea of BIGAN is as follows:

(1) Input real data $X$ and get it by encoder.

(2) Random noise $\mathrm{Z}$ is sampled from prior distribution and is obtained by generator.

(3) After the above two steps, the sum is obtained, and the two data pairs are input into the discriminator, so that the discriminator can distinguish whether the input data pairs are generated by the encoder or the generator.

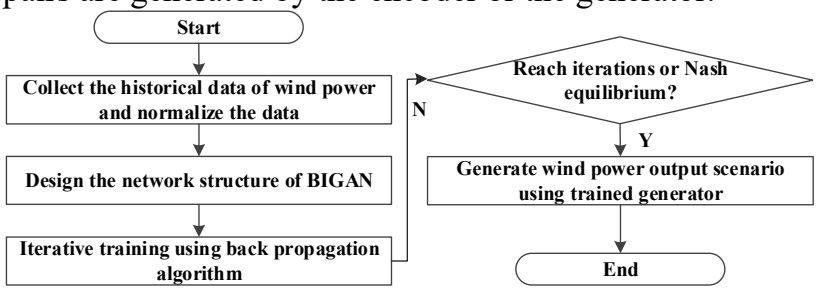

Figure 2. The flowchart of wind power output scenario generation

\subsection{Wind power scenario generation method based on BIGAN}

Different from image generation, the wind power data learned by Bigan is affected by physical factors such as atmosphere and spatial relationship of power generation units. However, the algorithm flow is similar. Firstly, the historical wind power data is input, and Bigan independently learns the statistical rules of the input data, and trains the encoder, generator and discriminator. After reaching or approaching the Nash equilibrium, the trained model is used to generate the data which accords with the statistical distribution characteristics of the original data. The process is shown in Figure 2.

\section{Multi-objective optimal planning of distribution network considering wind power uncertainty}

\subsection{Objective function}

In this paper, the system annual comprehensive cost $C$ and voltage offset $V_{D}$ are optimization objectives. The objective function is

$$
\min F=\min \left(C, V_{\mathrm{D}}\right)
$$

\subsubsection{System annual comprehensive cost}

$$
\begin{aligned}
& C=C_{\mathrm{C}}+C_{\mathrm{sOP}}+C_{\text {loss }} \\
& C_{\mathrm{C}}=\left(c_{1}+c_{2}\right) \sum_{i \in N_{\mathrm{c}}} Q_{\mathrm{c}, i}
\end{aligned}
$$

$c_{1}$ and $c_{2}$ are separately equipment cost and installation and construction cost of unit capacity capacitor. $N_{c}$ is the node set of the capacitors which are to be installed. $Q_{c, j}$ is the total capacity of the capacitor which is installed at node $i$.

$$
C_{\mathrm{SOP}}=\left(\frac{r(1+r)^{n}}{(1+r)^{n}-1}+\eta\right) \sum_{k=1}^{N_{\mathrm{SOP}}} c_{3} S_{\mathrm{SOP}, k}
$$

Where $r$ is the annual discount rate. $n$ is the useful life of SOP. $\eta$ is the coefficient of annual operation and maintenance cost. $N_{\mathrm{SOP}}$ is the number of the SOPs which are to be installed. $c_{3}$ is the investment cost of unit capacity. $S_{\mathrm{SOP}, k}$ is the installed capacity of the $k$ th SOP.

$$
C_{\text {loss }}=c_{4} \tau \sum_{s=1}^{N_{\mathrm{s}}} P_{\text {loss }}(s) \times p(s)
$$

Where $c_{4}$ is system unit price. $\tau$ is the maximum load utilization hour. $P_{\text {loss }}(s)$ is the active loss in the $s$ th scenario. $N_{S}$ is the number of scenarios.

\subsubsection{Voltage offset}

$$
V_{\mathrm{D}}=\max \left\{\left|U_{i}(s)-U_{N}\right| \| i=1, \mathrm{~L}, M, s=1, \mathrm{~L}, S\right\}
$$

Where $V_{D}$ is the maximum voltage offset in all scenarios. $M$ is the number of nodes. $U_{i}(s)$ is the voltage amplitude at node $i$ in the $s$ th scenario. $U_{N}$ is the rated voltage of line.

\section{2 constraint condition}

\subsubsection{Flow constraint}

$P_{i}(s)=U_{i}(s) \sum_{j=1}^{N} U_{j}(s)\left(G_{i j} \cos \theta_{i j}(s)+B_{i j} \sin \theta_{i j}(s)\right), Q_{i}(s)=U_{i}(s) \sum_{i=1}^{N} U_{j}(s)\left(G_{i j} \sin \theta_{i j}(s)+B_{i j} \cos \theta_{i j}(s)\right)$

3.2.2 Nodal voltage constraint

$$
U_{i}^{\min } \leq U_{i} \leq U_{i}^{\max }, i=1,2, \mathrm{~L} M
$$


3.2.3 Capacity constraint of capacitor installed on selected node

$$
Q_{\mathrm{c}, i}=n_{\mathrm{c}, i} q_{\mathrm{c}}, 0 \leq Q_{\mathrm{c}, i} \leq Q_{\mathrm{c}, i}^{\max }
$$

\subsubsection{Installation capacity constraint and operation} constraint of SOP

$$
S_{S O P, i j}=n_{S O P, i j} S_{S O P}, 0 \leq S_{S O P, i j} \leq S_{S O P, i j}^{\max }
$$

\subsubsection{Wind power operation constraint}

$$
P_{i}^{\mathrm{WT}}(s)=P_{\mathrm{C}}(s), Q_{i}^{\mathrm{WT}}(s)=P_{i}^{\mathrm{WT}}(s) \sqrt{\frac{1}{\cos ^{2} \varphi}-1}
$$

\section{Multi-objective optimization planning method of distribution network based on NSGA-II algorithm}

\subsection{Improved NSGA-II algorithm}

In order to solve the proposed non-linear multi-objective planning problem, the NSGA-II algorithm is used in this paper. Besides, three improvements are proposed, which are shown as follows.

\subsubsection{Adaptive hybrid crossover operator}

Relying on the simulated binary crossover (SBX) operator, the normal distribution crossover (NDX) operator is added. Both of the operators adaptively adjust the weights according the iterations according to (12). $p_{c}^{\min }, p_{c}^{\max }$ separately are the minimum crossover probability and maximum crossover probability of SBX. $i$ is the current iteration. gen is the total iteration.

$$
p_{\mathrm{c}}(i)=p_{\mathrm{c}}^{\min }+\left(p_{\mathrm{c}}^{\max }-p_{\mathrm{c}}^{\min }\right) \times i / \text { gen }
$$

\subsubsection{Adaptive mutation operator}

In order to avoid the situations, in which the optimization solution is destroyed or the worst solution is not improved. Only some poor individuals underwent polynomial mutation after the crossover operation. Firstly, the nondominated sorting is performed on the offspring. Then the mutation number is determined by mutation rate, which is varied by iteration and shown in (13). The mutation number of individuals in the current iteration is shown in (14).

$$
\begin{gathered}
p_{\mathrm{m}}(i)=p_{\mathrm{m}}^{\min }+\left(p_{\mathrm{m}}^{\max }-p_{\mathrm{m}}^{\min }\right) \times i / \text { gen } \\
n_{\text {variation }}=n_{\text {pop }} \times p_{\mathrm{m}}(i)
\end{gathered}
$$

\subsubsection{Improvement of elite retention strategy}

In this paper, the satisfaction of Pareto front is calculated in each iteration process, and the optimal solution of this iteration is obtained by using the optimal compromise strategy, and then retained. After the iteration, the optimal compromise strategy is used to select the optimal solution of the problem.

\subsection{Solving process of multi-objective optimization planning}

Combing random scenarios of wind power generated by BIGAN and the improved NSGA-II algorithm. The solving process of the proposed multi-objective planning model is shown in figure 3 .

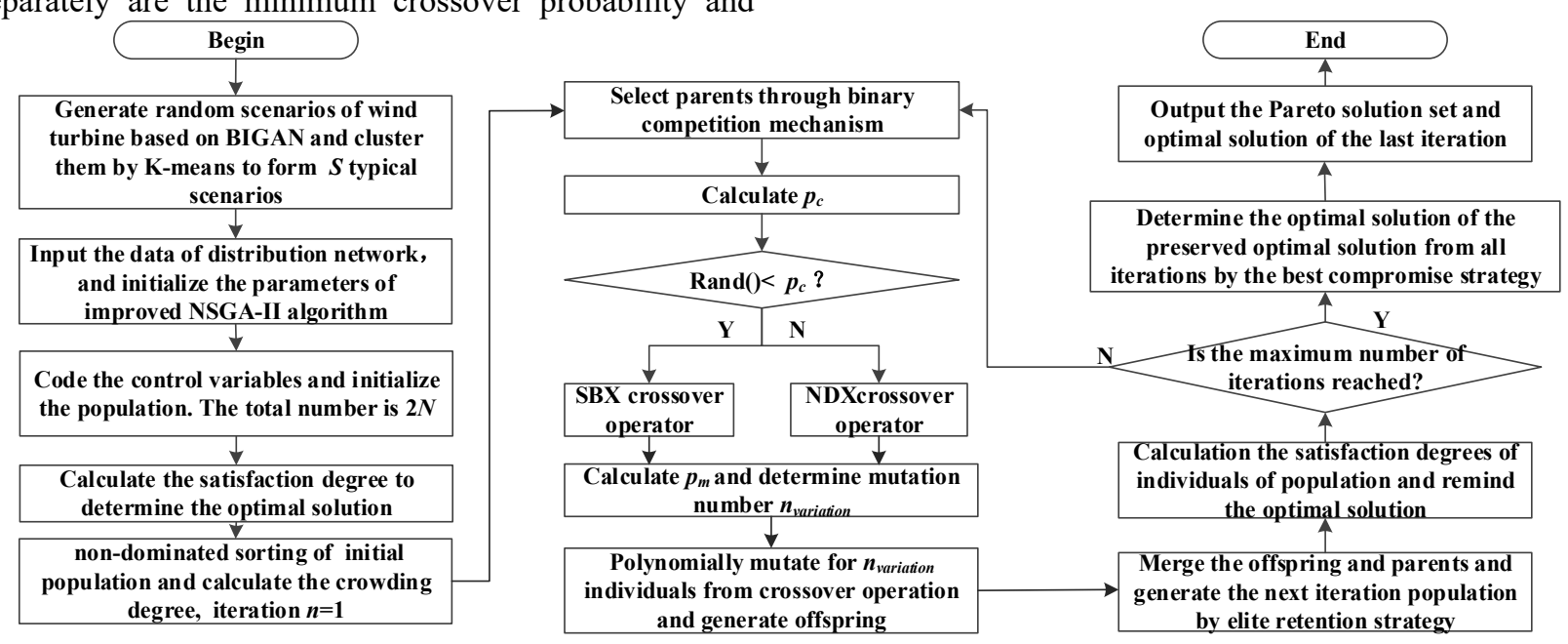

Figure 3. Solution process of optimal Planning model 


\section{Case study}

\subsection{System parameter}

In this paper, the improved IEEE 33 bus distribution network is selected for test ${ }^{1}$ and the topology is shown in Figure 4. Wind turbines WT1, WT2 and WT3 are separately connected at nodes 10,16 and 30, of which the power factor is 1.0. Assuming that the load of each node obeys normal distribution, the average load is the original system parameter value, and the variance is $10 \%$ of the average.

The shunt capacitors are to be installed at node 5, 11, $18,20,24,27$, and 31 . The maximum number of capacitor banks allowed to be installed for each selected node is 10 . The capacity of a single capacitor bank is $50 \mathrm{kvar}$. The cost of capacitor equipment is 30.8 yuan/kvar. The cost of capacitor installation and construction is 1.3 yuan $/ \mathrm{kvar}$. Considering the geographical location, the installation location of SOP is selected to the contact switch. The annual discount rate is 0.08 . The service life is 20 years. The investment cost per unit capacity is 1000 yuan $/ \mathrm{kVA}$. The unit optimized capacity is 100Kva. The annual operation and maintenance cost coefficient is 0.01 . The system electricity price is 0.5 yuan $/ \mathrm{kW} \cdot \mathrm{h}$. The annual maximum load utilization hours is 2000 hour.

The parameters of the algorithm are determined as follows. The population size is 200. The maximum number of iterations is 100 . The parameters $\eta$ of traditional NSGA-II algorithm using SBX operator and polynomial mutation operator are 20 , which is the same as the improved NSGA-II algorithm. Besides, $p_{c}^{\min }=0.1, p_{c}^{\max }$ $=0.9, p_{m}^{\min }=0.01, p_{m}^{\max }=0.2$. The size of Pareto front obtained by the two algorithms is 200 .

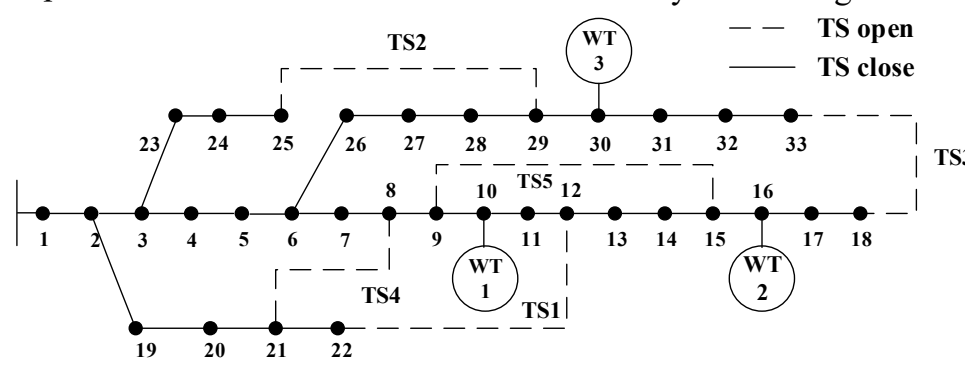

Figure 4. Improved IEEE 33 bus distribution network

\subsection{Generation of typical wind power scenarios}

In this paper, the actual data of three adjacent wind turbines in a certain area of the United States from January 1, 2012 to December 31, 2012 as the simulation data. $90 \%$ of the data are randomly selected as training set and the remaining $10 \%$ of the data as the test set ${ }^{6} .14400$ random scenarios are generated by BIGAN. After scene reduction by K-means clustering method, 20 typical wind power random scenes are formed, as shown in Table 1.

Table 1. Typical scenario of wind turbine

\begin{tabular}{cccccccccc}
\hline $\begin{array}{c}\text { Scen- } \\
\text { ario }\end{array}$ & WT1/kW & WT2/kW & WT3/kW & $\begin{array}{c}\text { Probab- } \\
\text { ility }\end{array}$ & Scen-ario & WT1/kW & WT2/kW & WT3/kW & $\begin{array}{c}\text { Probab- } \\
\text { ility }\end{array}$ \\
\hline 1 & 167.673 & 127.055 & 163.242 & $2.91 \%$ & 11 & 34.021 & 34.264 & 79.056 & $3.32 \%$ \\
2 & 52.38 & 74.39 & 69.39 & $3.89 \%$ & 12 & 8.107 & 7.324 & 8.445 & $16.7 \%$ \\
3 & 195.068 & 194.554 & 194.354 & $15.7 \%$ & 13 & 55.548 & 120.479 & 94.43 & $2.35 \%$ \\
4 & 26.023 & 66.968 & 32.747 & $4.87 \%$ & 14 & 128.574 & 163.535 & 159.83 & $3.48 \%$ \\
5 & 109.994 & 147.803 & 104.415 & $2.8 \%$ & 15 & 165.303 & 162.449 & 102.567 & $2.24 \%$ \\
6 & 56.216 & 31.595 & 29.846 & $4.95 \%$ & 16 & 18.305 & 29.351 & 15.677 & $7.98 \%$ \\
7 & 115.817 & 102.294 & 82.1 & $2.97 \%$ & 17 & 19.608 & 20.187 & 40.913 & $6.16 \%$ \\
8 & 75.836 & 134.168 & 144.265 & $2.32 \%$ & 18 & 175.432 & 17.524 & 170.457 & $7.16 \%$ \\
9 & 65.63 & 67.563 & 121.736 & $2.84 \%$ & 19 & 61.677 & 115.418 & 42.803 & $2.38 \%$ \\
10 & 101.062 & 52.798 & 57.72 & $2.38 \%$ & 20 & 129.963 & 88.027 & 133.974 & $2.66 \%$ \\
\hline
\end{tabular}

\subsection{Analysis of planning results}

In order to analyse the effect of different configuration strategies, the following four simulation schemes are set for comparative analysis. The definition of each simulation scheme is as follows: scheme 1, no optimal configuration; scheme 2, separate configuration of SOP; scheme 3, separate configuration of shunt capacitor; scheme 4, coordinated configuration of SOP and shunt capacitor. The improved NSGA-II algorithm proposed in this paper is used to solve the multi-objective optimization planning model, and the planning results of four simulation schemes are obtained, as shown in Table 2.

Compared with scheme 2, scheme 3 , and scheme 4, scheme 3 with shunt capacitor alone owns the smallest $C$, which is 149900 yuan. Meanwhile $C_{\text {loss }}$ is the largest, which is 11619000 yuan. In scheme 2 with SOP alone, $C$ is the largest, which is 2474800 yuan. $C_{\text {loss }}$ ranks second, which is 113260 yuan. In scheme 4 with SOP and shunt capacitor coordination, $C$ is between scheme 2 and scheme 3 , in which $C_{\text {loss }}$ is the smallest, and the voltage offset is the smallest. In conclusion, the coordinated configuration effect of SOP and shunt capacitor in scheme 4 is the best with the smallest $C_{\text {loss }}$ in all schemes, in which 
the annual comprehensive cost of the system is more economical, and the voltage quality is the highest.

Table 2. Planning results of four simulation schemes

\begin{tabular}{|c|c|c|c|c|c|}
\hline Scheme & $\begin{array}{l}\text { Node(Number of installed capacitor banks); } \\
\text { Switch position(installed capacity/kVA) }\end{array}$ & $\begin{array}{c}C_{C} / 10^{4} \mathrm{y} \\
\text { uan }\end{array}$ & $\begin{array}{c}C / 10^{4} \text { yuan } \\
C S O P / 10^{4} \mathrm{y} \\
\text { uan } \\
\end{array}$ & $\begin{array}{c}C_{\text {loss }} / 10^{4} \mathrm{yu} \\
\text { an }\end{array}$ & $\begin{array}{l}\text { Voltage } \\
\text { offset } / \mathrm{kV}\end{array}$ \\
\hline 1 & $\begin{array}{l}5(0), 11(0), 18(0), 20(0), 24(0), 27(0), 31(10) ; 12- \\
22(0), 25-29(0), 18-33(0), 8-21(0), 9-15(0)\end{array}$ & 0 & 17.409 & 17.409 & 1.092 \\
\hline 2 & $25-29(400), 18-33(800)$ & 0 & $\begin{array}{l}24.748 \\
13.422\end{array}$ & 11.326 & 0.555 \\
\hline 3 & $5(0), 11(3), 18(5), 20(0), 24(0), 27(3), 31(10)$ & 3.371 & $\begin{array}{c}14.99 \\
0\end{array}$ & 11.619 & 0.764 \\
\hline 4 & $11(3), 18(6), 27(6), 31(10) ; 12-22(400), 25-29(600)$ & 4.013 & $\begin{array}{l}23.157 \\
11.185\end{array}$ & 7.959 & 0.434 \\
\hline
\end{tabular}

According to the planning results in scheme 4, the node voltage in each scenario after planning is shown in Figure 5. It can be seen from the figure that the node voltage of each scenario is between 0.93p.u. and 1.0.p.u. after the planning is completed, which meets the voltage constraints, indicating that the configuration of SOP and shunt capacitor is reasonable and effective.

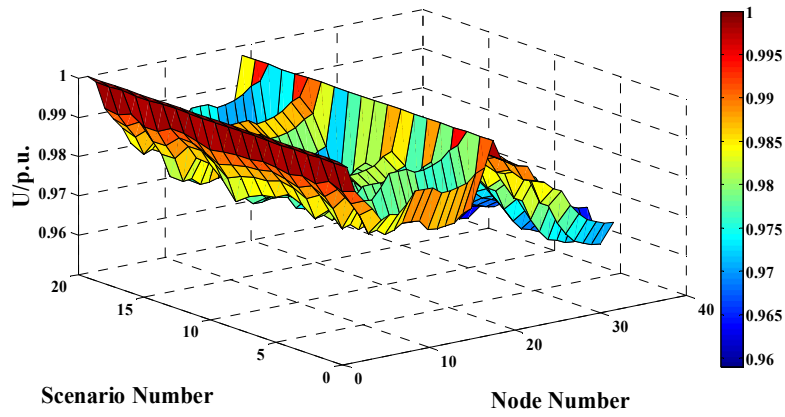

Figure 5. Node voltage of each scenario in Scheme 4

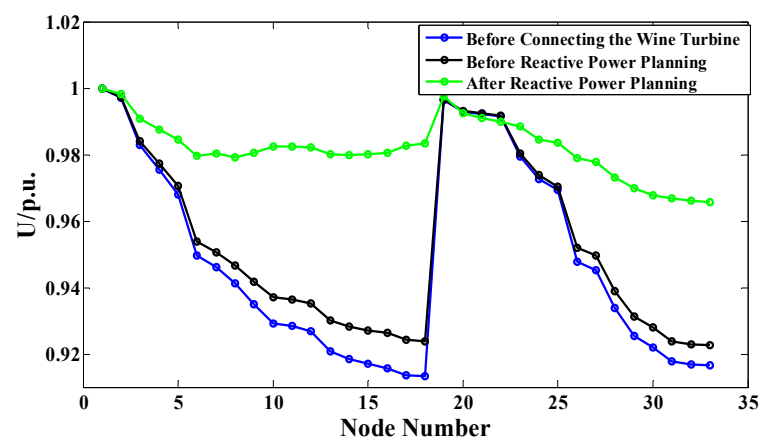

Figure 6. Node voltage changes before and after optimized configuration

To illustrate the planning effect of scheme 4, the comparison of node voltage expectation before and after planning is shown in Figure 6, and the economic analysis of planning results is shown in Table 3 .
Table 3. Economic analysis of planning results

\begin{tabular}{ccccc}
\hline Plan & $\begin{array}{c}C_{C}+C_{S O P} \\
/ 10^{4} \text { yuan }\end{array}$ & $\begin{array}{c}C_{\text {loss }} \\
/ 10^{4} \text { yuan }\end{array}$ & $C / 10^{4}$ yuan & $\begin{array}{c}\text { Voltage } \\
\text { offset } / \mathrm{kV}\end{array}$ \\
\hline 1 & 0 & 17.409 & 17.409 & 1.092 \\
4 & 15.198 & 7.959 & 25.639 & 0.434 \\
\hline
\end{tabular}

It can be seen from Figure 6 that before the wind turbine is connected, the node voltage level in the system is low, and the voltage of multiple nodes exceeds the lower limit. After the wind turbine is connected, the overall node voltage in the system is improved, and the voltage quality is improved. But it still exists the problem that the voltage of some nodes exceeds the lower limit. It can be seen from table 3 that after the optimal configuration, the SOP and the position and capacity of shunt capacitor are reasonably arranged, while the network active power loss is reduced and the voltage level of the system is improved. Among them, the voltage offset is reduced from $1.092 \mathrm{kV}$ to $0.434 \mathrm{kV}$. The network active power loss cost is reduced by 94500 yuan, of which the reduction ratio is $54.28 \%$. In conclusion, the economy of distribution system operation is improved.

\section{Conclusion}

Considering the uncertainty of wind power output, a multi-objective optimal planning model of distribution network is proposed based on scenario analysis method. Simulation analysis is carried out in the improved IEEE 33 bus system.

(1) The scenario generation model based on BIGAN has no explicit probability model and is completely datadriven, which accurately describes the uncertainty of wind power output.

(2) The simulation results of Pareto front show that compared with the traditional NSGA-II algorithm, the improved NSGA-II algorithm improves the optimization ability and convergence speed of the algorithm, ensures the diversity of the population, and makes the Pareto front 
distribution more uniform. Moreover, the Pareto front obtained in solving the multi-objective optimization programming model is closer to the coordinate axis, the distribution is more uniform, and the optimization effect is better.

(3) SOP and shunt capacitor bank complement each other, and the coordinated planning effect has the best effect, which significantly improves the voltage level of the distribution network and the economy of the system.

\section{Acknowledgments}

This work is supported in part by the State Grid Hengshui Electric Supply Company under Grant 5204HS20000J.

\section{References}

1. Wang S.X., Wang C.S. (2014) Morden distribution system analysis. Higher Education Press, Beijing.

2. Wang C.S., Song G.Y., Li P., et al. (2017) Optimal configuration of soft open point for active distribution network considering the characteristics of distributed generation. Proceedings of the CSEE, 37(07): 18891896.

3. Dong X.C., Sun Y. Y., Pu T. J. (2020) Day-ahead scenario generation of renewable energy based on conditional GAN. Proceedings of the CSEE, 40(17): 5527-5536.

4. Chen Y.Z., Wang Y.Q., Kirschen D., et al. (2018) Model-free renewable scenario generation using generative adversarial networks. IEEE Transactions on Power Systems,33(03): 3265-3275.

5. Liu B.L., Huang X.L., Li J., et al. (2016) MultiObjective Optimization of Active Distribution Network by Coordinating Energy Storage System and Flexible Load. Power System Technology, 40(05):1394-1399.

6. Bai J., Wang S.X., Zhao Q. Y., et al. (2020) Probabilistic load flow calculation for distribution network considering the uncertainty of wind power Output. Proceedings of the CSU-EPSA, 1-6[2020-0705]. https://doi.org/10.19635/j.cnki.csu-epsa.000455. 